\title{
Comparison of Thrombotic Events and Mortality in Patients with Community-Acquired Pneumonia and COVID-19: A Multicenter Observational Study
}

\author{
Roberto Cangemi ${ }^{1}$ Camilla Calvieri ${ }^{2}$ Marco Falcone ${ }^{3}$ Francesco Cipollone ${ }^{4}$ Giancarlo Ceccarelli ${ }^{5}$ \\ Pasquale Pignatelli ${ }^{2,6}$ Damiano D’Ardes ${ }^{4}$ Matteo Pirro $^{7}$ Francesco Alessandri ${ }^{8}$ Miriam Lichtner ${ }^{9}$ \\ Gabriella D’Ettorre ${ }^{5}$ Alessandra Oliva ${ }^{5}$ Raissa Aronica ${ }^{5}$ Monica Rocco ${ }^{10}$ Mario Venditti ${ }^{5}$ \\ Giulio Francesco Romiti ${ }^{10}$ Giusy Tiseo $^{3}$ Gloria Taliani ${ }^{1}$ Francesco Menichetti ${ }^{3}$ Francesco Pugliese ${ }^{8}$ \\ Claudio Maria Mastroianni ${ }^{5}$ Francesco Violi ${ }^{2,6}$
}

${ }^{1}$ Department of Translational and Precision Medicine, Sapienza University of Rome, Rome, Italy

2 Department of Clinical Internal, I Clinica Medica, Anaesthesiologic and Cardiovascular Sciences, Sapienza University of Rome, Rome, Italy

${ }^{3}$ Department of Clinical and Experimental Medicine, University of Pisa, Pisa, Italy

${ }^{4}$ Department of Medicine and Aging, “G. D’Annunzio" University of Chieti-Pescara, Chieti, Italy

${ }^{5}$ Department of Public Health and Infectious Diseases, Sapienza University of Rome, Rome, Italy

6 Mediterranea Cardiocentro, Naples, Italy

${ }^{7}$ Unit of Internal Medicine, Department of Medicine, University of Perugia, Perugia, Italy
Address for correspondence Francesco Violi, MD, I Clinica Medica, Sapienza University of Rome, Viale del Policlinico 155, Roma, 00161, Italy (e-mail: francesco.violi@uniroma1.it).

${ }^{8}$ Department of General Surgery Paride Stefanini, Sapienza University of Rome, Rome, Italy

${ }^{9}$ Infectious Diseases Unit, Santa Maria Goretti Hospital, Sapienza University of Rome, Latina, Italy

10 Department of Clinical and Surgical Translational Medicine, Sant' Andrea Hospital, Sapienza University of Rome, Rome, Italy

\section{Abstract \\ Keywords \\ - community-acquired pneumonia \\ - COVID-19 \\ - thrombosis}

Background It is still unclear if patients with community-acquired pneumonia (CAP) and coronavirus disease 2019 (COVID-19) have different rate, typology, and impact of thrombosis on survival.

Methods In this multicenter observational cohort study, 1,138 patients, hospitalized for CAP $(n=559)$ or COVID-19 $(n=579)$ from seven clinical centers in Italy, were included in the study. Consecutive adult patients (age $\geq 18$ years) with confirmed COVID-19-related pneumonia, with or without mechanical ventilation, hospitalized from March 1, 2020 to April 30, 2020, were enrolled. COVID-19 was diagnosed based on the World Health Organization interim guidance. Patients were followed-up until discharge or in-hospital death, registering the occurrence of thrombotic events including ischemic/embolic events.

Results During the in-hospital stay, $11.4 \%$ of CAP and 15.5\% of COVID-19 patients experienced thrombotic events $(p=0.046)$. In CAP patients all the events were arterial thromboses, while in COVID-19 patients $8.3 \%$ were venous and $7.2 \%$ arterial thromboses. received

May 25, 2021

accepted after revision

November 8, 2021

published online

November 10, 2021
(C) 2021. Thieme. All rights reserved. Georg Thieme Verlag KG,

Rüdigerstraße 14,

70469 Stuttgart, Germany
DOI https://doi.org/ 10.1055/a-1692-9939. ISSN 0340-6245. 
During the in-hospital follow-up, 3\% of CAP patients and 17\% of COVID-19 patients died $(p<0.001)$. The highest mortality rate was found among COVID-19 patients with thrombotic events ( 47.6 vs. $13.4 \%$ in thrombotic-event-free patients; $p<0.001)$. In CAP, $13.8 \%$ of patients experiencing thrombotic events died versus $1.8 \%$ of thrombotic event-free ones $(p<0.001)$. A multivariable Cox-regression analysis confirmed a higher risk of death in COVID-19 patients with thrombotic events (hazard ratio: 2.1; 95\% confidence interval: $1.4-3.3 ; p<0.001)$.

Conclusion Compared with CAP, COVID-19 is characterized by a higher burden of thrombotic events, different thrombosis typology and higher risk of thrombosisrelated in-hospital mortality.

\section{Introduction}

Coronavirus disease 2019 (COVID-19) is a pandemic characterized by severe acute respiratory syndrome coronavirus 2 (SARS-CoV-2) infection needing mechanical ventilation and intensive care unit (ICU) treatment. Among the factors predisposing to poor survival, thrombotic complications have been suggested to have an important role. ${ }^{1,2}$ Accordingly, clinical studies in COVID-19 showed a high incidence of venous and arterial thromboembolism, which was dependent upon a hypercoagulation state, as depicted by elevated plasma levels of D-dimer. ${ }^{3,4}$ This clinical feature resembles that of patients affected by community-acquired pneumonia (CAP), which is prevalently caused by viruses, ${ }^{5}$ and is among the commonest cause of hospitalization for pneumonia. ${ }^{5,6}$ Thus, patients hospitalized for CAP experience approximately $10 \%$ of thrombotic events, which are prevalently localized in the arterial vessels and are predictive of short- and long-term mortality. ${ }^{7,8}$ A previous study reported that venous thromboembolism is apparently higher in CAP versus COVID-19, ${ }^{9}$ while the intrahospital incidence of arterial thrombosis in CAP and COVID-19 needs to be clarified. Analysis of the different impacts of venous and arterial thromboses in CAP and COVID-19 may be relevant not only to provide insight into the pathophysiology of the two types of pneumonia but also to develop appropriate therapeutic strategies to lower the risk of thrombosis and eventually mortality. At this end, we performed an observational prospective study to compare predictors, rates of venous and arterial thromboses, and thrombosis-related mortality in CAP and COVID-19.

\section{Methods}

\section{Study Design and Population}

\section{CAP Patients}

We analyzed data from a prospective observational study aimed to evaluate the incidence of major vascular events in hospitalized adult patients with pneumonia (clinical.trial. gov: NCT01773863).

This cohort study prospectively recruited and followed up consecutive patients referred to three medical centers from the University-Hospital Policlinico Umberto I, Sapienza Uni- versity of Rome, Italy (Department of Internal Medicine and Medical Specialties, Department of Clinical Medicine, Department of Public Health and Infectious Diseases).

We consecutively enrolled 559 adults who met the following criteria: (1) age $\geq 18$ years; (2) clinical presentation of an acute illness with at least two or more of the signs or symptoms of CAP, as previously reported ${ }^{10}$; and (3) presence of new consolidation(s) on chest X-ray. ${ }^{6}$ Pneumonia was defined as CAP diagnosed upon hospitalization in a patient who did not meet the criteria for health care-associated pneumonia. ${ }^{11}$ Exclusion criteria were: radiographic evidence of pre-existing infiltrates; immunosuppression (human immunodeficiency virus infection, chemotherapy, high dose of immunosuppressive agents to prevent the rejection of transplanted organs and tissues or to treat autoimmune diseases); presence of malignancy; pregnancy or breastfeeding; documented severe allergy to antibiotics; health careassociated pneumonia. ${ }^{11}$

All patients with CAP admitted to the three units after giving written informed consent from October 2011 to January 2016 were prospectively recruited and followed up. The study was conducted according to the principles stated in the Declaration of Helsinki and approved by the local ethics committee.

\section{COVID-19 Patients}

Patients hospitalized for COVID-19-related pneumonia were recruited in seven Italian hospitals from Rome (two centers), Latina, Firenze, Perugia, Pisa, and Chieti.

We included consecutive adult patients (age $\geq 18$ years) with confirmed COVID-19-related pneumonia, with or without mechanical ventilation, hospitalized from March 1, 2020 to April 30, 2020. COVID-19 was diagnosed on the basis of the World Health Organization interim guidance. A confirmed case was defined as a person with laboratory confirmation of COVID-19 infection, irrespective of clinical signs and symptoms. Oropharyngeal and nasopharyngeal swabs for laboratory diagnosis of COVID-19 were performed in duplicate: SARS- Cov2 E and $S$ gene were detected by a reverse transcriptase polymerase chain reaction.

High-resolution computed tomography scan was used to identify lung involvement according to the Official Diagnosis and Treatment Protocol (6th edition) declared by the National 
Health Commission of China. Typical CT findings of SARS-CoV2related pneumonia were considered: consolidation, groundglass opacities, crazy paving, and/or reticular pattern. ${ }^{12}$

Radiologic abnormalities were reviewed by attending physicians in respiratory medicine who extracted the data. Major disagreement between two reviewers was resolved by consultation with a third reviewer. Ethical approval for this study was obtained from Ethics Committee of Azienda Ospedaliero Universitaria Policlinico Umberto I (approval number/ID Prot. 109/2020).

\section{Baseline Assessment}

Demographic, clinical, laboratory, and radiological results were extracted from electronic medical records of patients enrolled. Routine laboratory analysis included D-dimer, high-sensitivity C-reactive protein (hs-CRP), serum albumin and was collected at hospital admission. Prevalence of comorbidities such as diabetes mellitus, hypertension, coronary artery disease (CAD), heart failure (HF), chronic kidney disease, and chronic obstructive pulmonary disease (COPD) were recorded as previously described. ${ }^{10}$

The CURB-65 (confusion, uremia, respiratory rate, blood pressure, age $\geq 65$ years) score, a validated pneumonia severity assessment tool, ${ }^{13}$ was calculated based on clinical admission and laboratory parameters.

Data about therapeutic treatments were collected at hospital admission.

\section{Assessment of In-Hospital Thrombotic Events}

Patients were followed-up until discharge or in-hospital death. We registered the occurrence of thrombotic events including ischemic/embolic events, which were categorized as follows: pulmonary embolism (detected by lung CT pulmonary angiogram with intravenous contrast), ${ }^{14}$ acute myocardial infarction (diagnosed on the basis of the diagnostic electrocardiographic findings associated with elevation of serum markers of myocardial necrosis), ${ }^{15}$ acute ischemic strokes (identified by observing the onset of new focal neurological signs and symptoms and confirmed with magnetic resonance imaging or CT imaging), ${ }^{16}$ acute limb ischemia diagnosed according to guidelines, ${ }^{17}$ superficial/peripheral vein thrombosis and deep venous thrombosis diagnosed as previously described, ${ }^{18}$ and unusual vein thrombosis detected in symptomatic patients by CT scan (i.e., gonadic vein thrombosis, splanchnic venous thrombosis).

Diagnostic procedures were performed upon attending physician's requests.

\section{Statistical Analysis}

Categorical variables are reported as counts and percentages and continuous variables as mean \pm standard deviation, or medians and interquartile ranges. Differences between percentages were assessed by chi-square or Fisher exact tests. All continuous variables were tested for normality with the Shapiro-Wilk test. Student's unpaired $t$-tests were used for normally distributed continuous variables. Appropriate nonparametric tests (Mann-Whitney and Spearman rank correlation tests) were used for the other variables. The bivariate and multivariate effects of prognostic factors on thrombotic events were assessed by means of logistic regression models. Nonnormally distributed continuous variables were log-transformed before entering the model. Wald confidence intervals (CIs) and tests for odds ratios (OR) and adjusted OR were computed on the basis of the estimated standard errors. Survival curves were estimated using the Kaplan-Meier product limit estimator and compared using the log-rank test. Cox proportional hazards analysis was used to calculate the adjusted hazard ratios (HRs) and $95 \% \mathrm{CI}$ for each clinical variable. In the multivariable models, a random intercept, accounting for the recruiting centers, was also added to evaluate the possible effects of the different centers on the outcomes. Only $p$-values $<0.05$ were considered statistically significant. All tests were two-tailed, and analyses were performed using computer software packages (IBM SPSS Statistics 25).

\section{Results}

The study included 1,138 patients, hospitalized for CAP ( $n=559,212$ females; 347 males; age: $71.9 \pm 16.2$ years) or COVID-19 $(n=579 ; 261$ females; 318 males; age $65.3 \pm 16.6$ years). Clinical and laboratory characteristics of CAP and COVID-19 patients are reported in - Table 1.

CAP patients were older and with a higher prevalence of males. Moreover, a higher prevalence of comorbidities was found among CAP patients; thus, CAP patients were more likely to have $C A D$, diabetes, hypertension, atrial fibrillation (AF), and HF than COVID-19 ones. White blood cells (WBCs) and platelet count were higher in CAP patients. During the inhospital stay, CAP patients were more likely to be treated with antiplatelet or oral anticoagulants, while COVID-19 patients were prevalently treated with heparins ( - Table 1). Overall, at admission, no significant differences in CURB-65 score was found between CAP and COVID patients.

Among the 579 COVID-19 patients, 80 (14\%) patients were admitted to ICU. ICU patients showed lower prevalence of female sex, higher levels of hs-CRP, D-dimer, and WBC, and lower serum albumin and rate of sPO2 than non-ICU ones. The CURB-65 score was higher in ICU patients than non-ICU COVID-19 or CAP ones (- Table 1). Hospital stay length was 10 [7-13] days for CAP patients and 19 [12-27] for COVID-19 patients $(p<0.001)$.

\section{Thrombotic Complications}

During the in-hospital stay, 64 CAP patients (11.4\%) and 90 COVID-19 (15.5\%) patients experienced a thrombotic event $(p=0.046)$. In CAP patients all the events were arterial thromboses, while in COVID-19 patients 48 (8.3\%) were venous and 42 (7.2\%) arterial thromboses (-Table 2). Among COVID-19 patients, a greater incidence of both venous and arterial thromboses was found in those admitted to ICU (overall incidence of thrombosis in ICU vs. non-ICU patients: 41.3 vs. $11.4 \%$, respectively; $p<0.001$ ). Separate analysis of thrombotic events in non-ICU COVID-19 versus CAP showed no significant difference; however, a higher incidence of venous thrombosis and a lower incidence of arterial thrombosis were detected in non-ICU COVID-19 versus CAP patients, respectively ( $\mathbf{- T a b l e ~} \mathbf{3}$ ). 
Table 1 Clinical and laboratory characteristics of CAP and COVID-19 patients at hospital admission

\begin{tabular}{|c|c|c|c|c|c|c|}
\hline & CAP & COVID-19 & $p$-Value & Non-ICU COVID-19 & ICU COVID-19 & $p$-Value \\
\hline No. & 559 & 579 & & 499 & 80 & \\
\hline Age $(y)$ & $71.9 \pm 16.2$ & $65.3 \pm 16.6$ & $<0.001$ & $65.1 \pm 16.9$ & $66.2 \pm 14.0$ & 0.564 \\
\hline Female sex & $38 \%$ & $45 \%$ & 0.026 & $49 \%$ & $19 \%$ & $<0.001$ \\
\hline ICU & 0 & $14 \%$ & $<0.001$ & - & - & - \\
\hline Smoker & $20 \%$ & $16 \%$ & 0.089 & $16 \%$ & $20 \%$ & 0.417 \\
\hline CAD & $30 \%$ & $17 \%$ & $<0.001$ & $17 \%$ & $12 \%$ & 0.415 \\
\hline COPD & $32 \%$ & $12 \%$ & $<0.001$ & $11 \%$ & $19 \%$ & 0.063 \\
\hline Diabetes & $26 \%$ & $17 \%$ & $<0.001$ & $16 \%$ & $22 \%$ & 0.151 \\
\hline Hypertension & $72 \%$ & $48 \%$ & $<0.001$ & $48 \%$ & $55 \%$ & 0.279 \\
\hline Paroxysmal AF & $13 \%$ & $6 \%$ & $<0.001$ & $6 \%$ & $7 \%$ & 0.617 \\
\hline Chronic AF & $14 \%$ & $4 \%$ & $<0.001$ & $5 \%$ & $0 \%$ & 0.080 \\
\hline Chronic heart failure & $22 \%$ & $15 \%$ & 0.007 & $15 \%$ & $13 \%$ & 0.614 \\
\hline sPO2 (\%) & $93.3 \pm 6.9$ & $94.0 \pm 6.2$ & 0.158 & $94.2 \pm 5.9$ & $91.2 \pm 8.8$ & 0.012 \\
\hline WBC (No. $/ \mathrm{mm}^{3}$ ) & $\begin{array}{l}9,900 \\
{[7,480-13,540]}\end{array}$ & $\begin{array}{l}6,010 \\
{[4,560-8,350]}\end{array}$ & $<0.001$ & $5,745[4,400-7,345]$ & $8,610[5,560-12,250]$ & $<0.001$ \\
\hline $\operatorname{PLT}\left(\mathrm{n} / \mathrm{mm}^{3}\right) \times 10^{3}$ & $233[179-300]$ & 193 [156-246] & $<0.001$ & 193 [157-243] & $193[141-288]$ & 0.742 \\
\hline $\begin{array}{l}\text { Serum creatinine } \\
(\mathrm{mg} / \mathrm{dL})\end{array}$ & $1.20 \pm 0.83$ & $1.10 \pm 0.86$ & 0.081 & $1.09 \pm 0.91$ & $1.13 \pm 0.57$ & 0.699 \\
\hline Hs-CRP (mg/L) & $48[15-130]$ & 53 [20-129] & 0.409 & 48 [19-117] & $97[30-210]$ & 0.008 \\
\hline Albumin $(\mathrm{g} / \mathrm{L})$ & $35[31-38]$ & $35[31-39]$ & 0.484 & 36 [32-39] & $31[27-35]$ & $<0.001$ \\
\hline D-dimer (ng/mL) & $\begin{array}{l}1,182 \\
{[547-2,377]}\end{array}$ & $\begin{array}{l}939 \\
{[466-2,022]}\end{array}$ & 0.423 & $785[420-1,690]$ & $2,254[1,051-4,610]$ & $<0.001$ \\
\hline Antiplatelet drugs & $40 \%$ & $18 \%$ & $<0.001$ & $14 \%$ & $30 \%$ & 0.102 \\
\hline OACs & $14 \%$ & $6 \%$ & $<0.001$ & $7 \%$ & $5 \%$ & 1.000 \\
\hline Heparins & $6 \%$ & $49 \%$ & $<0.001$ & $38 \%$ & $98 \%$ & $<0.001$ \\
\hline CURB-65 & $1.76 \pm 1.09$ & $1.79 \pm 1.40$ & 0.667 & $1.70 \pm 1.41$ & $2.35 \pm 1.2$ & $<0.001$ \\
\hline
\end{tabular}

Abbreviations: AF, atrial fibrillation; CAD, coronary artery disease; CAP, community-acquired pneumonia; COPD, chronic obstructive pulmonary disease, hs-CRP, high sensitivity C-reactive protein; ICU, intensive care unit; OACs, oral anticoagulants; PLT, platelets; WBC, white blood cell. Note: Data are expressed as percentages, mean $\pm \mathrm{SD}$, or median [interquartile range].

Patients' baseline characteristics according to in-hospital thrombotic complications are represented in - Table 4. CAP patients who experienced a thrombotic event were older, more likely to have CAD, HF, and hypertension than thrombotic-event-free ones. COVID-19 patients who experienced a thrombotic event were older and more likely to have HF and to be admitted to ICU than thrombotic-event-free ones; moreover, they showed higher levels of WBC and D-dimer, and lower serum albumin (-Table 4 ).

In logistic regression analyses, thrombotic events were independently associated with age, HF, CAD, and the CURB65 score in CAP patients; ICU admission, age, and D-dimer in COVID-19 patients; and to ICU admission, age, D-dimer, CAD, and HF in the whole cohort (-Table 5).

\section{Mortality}

During the in-hospital follow-up, 17 CAP patients (3\%) and 98 COVID-19 patients $(17 \%)$ died $(p<0.001)$; among nonsurvivor COVID-19 patients, 62 out of the 499 (12.4\%) were
non-ICU and 36 out of 80 (45\%) were ICU patients $(p<0.001$; - Fig. 1A).

CAP patients who died were older than those who survived. COVID-19 patients who died were older, more likely to be male, and had higher prevalence of comorbidities like CAD, COPD, diabetes, hypertension, paroxysmal AF, and HF than the survivor ones. Moreover, nonsurvivor COVID-19 patients had significant lower baseline sPO2 and serum albumin and higher WBC, hs-CRP, and D-dimer; also, they more frequently needed ICU than the survivor ones ( - Table 6 ).

The occurrence of thrombotic events was associated with an increased risk of death in both CAP and COVID-19 patients. In CAP patients, $13.8 \%$ of patients experiencing thrombotic events died versus $1.8 \%$ of thrombotic-event-free ones (hazard ratio: $3.4 ; 95 \% \mathrm{CI}: 1.2-9.3 ; p=0.018)$; while in COVID-19 patients, $47.6 \%$ of patients experiencing thrombotic events died versus $13.4 \%$ of thrombotic-event-free ones (-Fig. 1B).

Multivariable Cox regression analyses showed that, in COVID-19 patients, thrombotic events, older age, COPD, 
Table 2 In-hospital thrombotic events in CAP and COVID-19 patients

\begin{tabular}{|c|c|c|c|}
\hline & CAP $(n=559)$ & COVID-19 $(n=579)$ & $p$-Value \\
\hline \multicolumn{4}{|l|}{ Outcomes } \\
\hline Superficial vein thrombosis & $0 \%$ & $10(1.7 \%)$ & \\
\hline DVT & $0 \%$ & $16(2.8 \%)$ & $<0.001$ \\
\hline Pulmonary embolism & $0 \%$ & $18(3.1 \%)$ & \\
\hline Unusual vein thrombosis & $0 \%$ & $4(0.7 \%)$ & \\
\hline Total venous thrombosis & $0 \%$ & $48(8.3 \%)$ & $<0.001$ \\
\hline Peripheral ischemic disease & $0(0 \%)$ & $9(1.6 \%)$ & \\
\hline Myocardial infarction & $52(9.3 \%)$ & $21(3.6 \%)$ & \\
\hline TIA/stroke & $12(2.1 \%)$ & $12(2.1 \%)$ & \\
\hline Total arterial thrombosis & $64(11.4 \%)$ & $42(7.2 \%)$ & 0.019 \\
\hline Total thrombosis & 64 (11.4\%) & $90(15.5 \%)$ & 0.046 \\
\hline
\end{tabular}

Abbreviations: CAP, community-acquired pneumonia; DVT, deep venous thrombosis; TIA, transient ischemic attack.

Table 3 In-hospital thrombotic events in CAP, ICU, and non-ICU COVID-19 patients

\begin{tabular}{|c|c|c|c|c|c|}
\hline & CAP & Non-ICU COVID-19 & ICU-Covid-19 & $\begin{array}{l}p \text {-Value }{ }^{*} \\
\text { (CAP vs. } \\
\text { non-ICU COVID-19) }\end{array}$ & $\begin{array}{l}\text { p-Value* } \\
\text { (non-ICU vs. ICU } \\
\text { COVID-19) }\end{array}$ \\
\hline$N$ & 559 & 499 & 80 & & \\
\hline \multicolumn{6}{|l|}{ Outcomes } \\
\hline Superficial vein thrombosis & $0(0 \%)$ & $6(1.2 \%)$ & $4(5.0 \%)$ & & \\
\hline DVT & $0(0 \%)$ & $11(2.2 \%)$ & $5(6.3 \%)$ & & \\
\hline Pulmonary embolism & $0(0 \%)$ & $6(1.2 \%)$ & $12(15 \%)$ & & \\
\hline Unusual vein thrombosis & $0(0 \%)$ & $3(0.6 \%)$ & $1(1.3 \%)$ & & \\
\hline Total venous thrombosis & $0(0 \%)$ & $26(5.2 \%)$ & $22(27.5 \%)$ & $<0.001$ & $<0.001$ \\
\hline Peripheral ischemic disease & $0(0 \%)$ & $4(0.8 \%)$ & $5(6.3 \%)$ & & \\
\hline Myocardial infarction & $52(9.3 \%)$ & $16(3.2 \%)$ & $5(6.3 \%)$ & & \\
\hline TIA/stroke & $12(2.1 \%)$ & $11(2.2 \%)$ & $1(1.3 \%)$ & & \\
\hline Total arterial thrombosis & 64 (11.4\%) & $31(6.2 \%)$ & $11(13.8 \%)$ & 0.012 & 0.029 \\
\hline Total thrombosis & 64 (11.4\%) & $57(11.4 \%)$ & $33(41.3 \%)$ & 0.989 & $<0.001$ \\
\hline
\end{tabular}

Abbreviations: CAP, community-acquired pneumonia; DVT, deep venous thrombosis; TIA, transient ischemic attack.

baseline values of sPO2, and serum albumin were independently associated with death and that, in the whole cohort, COVID-19 was associated with an increased risk of mortality (-Table 7).

\section{Discussion}

The study provides evidence that, compared with CAP, COVID-19 is associated with a higher burden of thrombotic events and different thrombosis types. Furthermore, the intra-hospital mortality risk is roughly fivefold higher in COVID-19 compared with CAP.

Our analysis displays relevant differences in terms of clinical characteristics at admission and clinical outcomes including thrombotic events and mortality between two populations of patients hospitalized for CAP or COVID-19. Thus, at admission to the hospital, CAP population was older, more frequently male, and with a higher burden of cardiovascular and pulmonary disease; among the laboratory variables CAP showed higher WBC and platelet count. Data regarding systemic inflammation and clotting activation did not show any difference between the two populations; however, separate analysis of ICU and not-ICU revealed a significant difference between the two populations, with ICU COVID-19 patients showing higher values of hs-CRP and Ddimer versus non-ICU ones and CAP. Concerning the antithrombotic drugs, antiplatelet treatment was more frequently used in CAP, while anticoagulants were more frequently used in COVID-19, especially in those needing ICU. It is interesting to note, however, that the number of COVID-19 patients 
Table 4 Patients' baseline characteristics according to in-hospital thrombotic complications in CAP ad COVID-19 patients

\begin{tabular}{|c|c|c|c|c|c|c|}
\hline & CAP patients & & & COVID patients & & \\
\hline & $\begin{array}{l}\text { Without thrombotic } \\
\text { event }\end{array}$ & $\begin{array}{l}\text { With thrombotic } \\
\text { event }\end{array}$ & $P$-Value & $\begin{array}{l}\text { Without thrombotic } \\
\text { event }\end{array}$ & $\begin{array}{l}\text { With thrombotic } \\
\text { event }\end{array}$ & $p$-Value \\
\hline N & 495 & 64 & & 481 & 90 & \\
\hline Age & $70.7 \pm 16.5$ & $80.6 \pm 8.9$ & $<0.001$ & $64.1 \pm 16.8$ & $71.5 \pm 13.8$ & $<0.001$ \\
\hline Male sex & $62 \%$ & $62 \%$ & 0.941 & $54 \%$ & $62 \%$ & 0.168 \\
\hline ICU & n.a. & n.a. & n.a. & $11 \%$ & $59 \%$ & $<0.001$ \\
\hline Smoker & $20 \%$ & $17 \%$ & 0.661 & $16 \%$ & $16 \%$ & 0.849 \\
\hline CAD & $27 \%$ & $52 \%$ & $<0.001$ & $15 \%$ & $23 \%$ & 0.104 \\
\hline COPD & $32 \%$ & $33 \%$ & 0.885 & $12 \%$ & $14 \%$ & 0.710 \\
\hline Diabetes & $24 \%$ & $37 \%$ & 0.020 & $16 \%$ & $18 \%$ & 0.631 \\
\hline Hypertension & $70 \%$ & $84 \%$ & 0.016 & $48 \%$ & $57 \%$ & 0.123 \\
\hline Paroxysmal AF & $12 \%$ & $19 \%$ & 0.122 & $6 \%$ & $10 \%$ & 0.200 \\
\hline Chronic AF & $13 \%$ & $17 \%$ & 0.486 & $4 \%$ & $6 \%$ & 0.750 \\
\hline Chronic heart failure & $19 \%$ & $42 \%$ & $<0.001$ & $14 \%$ & $23 \%$ & 0.041 \\
\hline sPO2 (\%) & $93.6 \pm 7.1$ & $92.0 \pm 5.9$ & 0.879 & $94.4 \pm 6.0$ & $91.1 \pm 6.7$ & $<0.001$ \\
\hline$W B C\left(n / m^{3}\right)$ & $\begin{array}{l}9,890 \\
{[7,380-13,300]}\end{array}$ & $\begin{array}{l}10,522 \\
{[8,462-14,777]}\end{array}$ & 0.092 & $\begin{array}{l}5,870 \\
{[4,480-8,084]}\end{array}$ & $\begin{array}{l}7,330 \\
{[4,720-10,465]}\end{array}$ & 0.025 \\
\hline Hs-CRP (mg/L) & 48 [16-130] & 42 [13-108] & 0.552 & $48[20-122]$ & 65 [20-169] & 0.103 \\
\hline Albumin $(\mathrm{g} / \mathrm{L})$ & $35[31-38]$ & $35[32-38]$ & 0.824 & 36 [32-39] & $32[28-36]$ & $<0.001$ \\
\hline D-dimer (ng/mL) & $\begin{array}{l}1,115 \\
{[543-2,276]}\end{array}$ & $\begin{array}{l}1,464 \\
{[560-3,847]}\end{array}$ & 0.271 & $\begin{array}{l}828 \\
{[440-1,660]}\end{array}$ & $\begin{array}{l}1,972 \\
{[755-4,610]}\end{array}$ & $<0.001$ \\
\hline CURB-65 score & $1.68 \pm 1.0$ & $2.39 \pm 0.99$ & $<0.001$ & $1.73 \pm 1.25$ & $2.11 \pm 1.15$ & 0.005 \\
\hline
\end{tabular}

Abbreviations: AF, atrial fibrillation; CAD, coronary artery disease; CAP, community-acquired pneumonia; COPD, chronic obstructive pulmonary disease, hs-CRP, high-sensitivity C-reactive protein; ICU, intensive care unit; OACs, oral anticoagulants; PLT, platelets; sPO2, oxygen saturation; WBC, white blood cell.

Note: Data are expressed as percentages, mean \pm SD, or median [interquartile range].

treated with anticoagulants was relatively low, which may be consistent with the fact that, at the time of inclusion of our population, the relationship between COVID-19, thrombosis, and death was still unclear.

During the follow-up, COVID-19 patients experienced significantly more thrombotic events compared with CAP ones ( 15.5 vs. $11.4 \%$, respectively). Thrombosis typology was also different as CAP was essentially complicated by arterial ischemia such as myocardial infarction and stroke while no symptomatic venous thrombosis was detected. The low venous thrombosis incidence in CAP is in agreement with our previous report of a prospective multicenter study including more than 1,000 CAP patients, ${ }^{8}$ showing the absence of in-hospital DVT. However, this finding is apparently in contrast with a recent study by Mei et al, who reported $3.9 \%$ of venous thromboembolism in a retrospective analysis of 360 patients affected by CAP. ${ }^{9}$ The lack of information regarding diagnostic work-up and clinical presentation (symptomatic vs. asymptomatic) makes difficult a comparison with our report. As diagnostic work-up for venous thrombosis was not routinely performed, we cannot exclude that CAP could be complicated by asymptomatic venous thrombosis. This is a crucial methodological issue as we have previously shown that in acutely ill medical patients, including patients with $\mathrm{CAP},{ }^{19}$ venous thromboses are essentially asymptomatic and detectable at admission more than during the intra-hospital stay.

At variance with CAP, COVID-19 population experienced thrombosis in both artery and venous circulation with an equivalent incidence rate. Separate analysis of COVID-19 population revealed, however, that the burden of thrombotic events was much higher in ICU versus non-ICU with both venous and arterial thrombosis being at least twofold more frequent in ICU, suggesting that disease severity is a prerequisite for the occurrence of thrombosis. The multivariable logistic regression suggested that in CAP patients baseline cardiovascular comorbidities play a synergistic role in increasing thrombotic risk, while in COVID-19 patients the severity of the infectious disease, as depicted by ICU admission, and D-dimer levels at admission seem to play a more relevant role in thrombotic risk.

The difference in the incidence and typology of thrombosis in CAP versus COVID-19 suggests also that the two infections may display different mechanisms of thrombosis. It is noteworthy, for instance, that we did not find any difference of D-dimer in CAP with and without thrombosis, 
Thrombotic Events in CAP and COVID-19 Cangemi et al. 263

Table 5 Variables associated to thrombotic events in CAP and COVID-19 patients

\begin{tabular}{|c|c|c|c|c|}
\hline & \multirow[t]{2}{*}{ OR } & \multicolumn{2}{|l|}{$95 \% \mathrm{Cl}$} & \multirow[t]{2}{*}{$p$-Value } \\
\hline & & Lower & Upper & \\
\hline \multicolumn{5}{|l|}{$\mathrm{CAP}^{\mathrm{a}}$} \\
\hline Age $(y)^{b}$ & 1.04 & 1.01 & 1.07 & 0.011 \\
\hline Heart failure & 2.21 & 1.16 & 4.21 & 0.016 \\
\hline CAD & 1.92 & $1-04$ & 3.56 & 0.037 \\
\hline CURB-65 score ${ }^{\mathrm{b}}$ & 1.47 & 1.05 & 2.07 & 0.026 \\
\hline \multicolumn{5}{|l|}{ COVID-19 } \\
\hline ICU-admission & 4.14 & 2.34 & 7.34 & $<0.001$ \\
\hline Age $(y)^{b}$ & 1.02 & 1.01 & 1.04 & 0.013 \\
\hline $\log (\mathrm{D}-\mathrm{DIMER})^{\mathrm{b}}$ & 4.34 & 2.69 & 8.29 & $<0.001$ \\
\hline \multicolumn{5}{|l|}{ All patients ${ }^{d}$} \\
\hline ICU-admission & 5.60 & 2.34 & 9.52 & $<0.001$ \\
\hline Age $(y)^{b}$ & 1.03 & 1.01 & 1.04 & $<0.001$ \\
\hline Log (D-dimer) ${ }^{\mathrm{b}}$ & 3.55 & 2.16 & 5.87 & $<0.001$ \\
\hline CAD & 1.70 & 1.09 & 2.65 & 0.018 \\
\hline Heart failure & 1.75 & 1.10 & 2.78 & 0.017 \\
\hline
\end{tabular}

Abbreviations: $C A D$, coronary artery disease; $C A P$, community-acquired pneumonia; $\mathrm{Cl}$, confidence interval; OR, odd ratio.

${ }^{\mathrm{a} A f t e r}$ adjusting for sex, diabetes, arterial hypertension, chronic obstructive pulmonary disease, atrial fibrillation, smoking habit, hs-CRP, albumin, D-dimer, creatinine, aspirin, anticoagulant use, and the recruitment centers.

${ }^{b}$ For each increasing unit.

'After adjusting for sex, diabetes, arterial hypertension, CAD, heart failure, chronic obstructive pulmonary disease, atrial fibrillation, smoking habit, hs-CRP, albumin, aspirin and anticoagulant use, and the recruitment centers.

${ }^{\mathrm{d}}$ After adjusting for sex, diabetes, arterial hypertension, chronic obstructive pulmonary disease, atrial fibrillation, smoking habit, hs-CRP, albumin, creatinine, aspirin, anticoagulant use, and the recruitment centers.

while D-dimer was significantly associated with thrombotic events in COVID-19, suggesting a major role for clotting activation in this last setting. Consistent with this, hypofibrinolysis and low fibrinogen levels have been associated with hypercoagulation and pulmonary thromboembolism. ${ }^{20,21}$ This finding would imply a different pattern of thrombotic risk, with COVID-19 being more prone to be associated with factors eliciting clotting activation and eventually thrombosis; conversely, the typology of thrombosis (essentially arterial) would suggest a major role for platelet activation as a factor precipitating thrombosis in CAP. ${ }^{22}$ Further studies are necessary to elucidate this issue.

A relevant difference between CAP and COVID-19 also regarded the mortality risk, which was approximately five times higher in COVID-19 (17\%) compared with CAP (3.1\%); this difference, was, however, greatly influenced by COVID19 disease severity. Thus, overall survival analysis showed an early curve divergence between CAP and ICU-COVID-19, while the divergence was less impressive, but still significant,
A

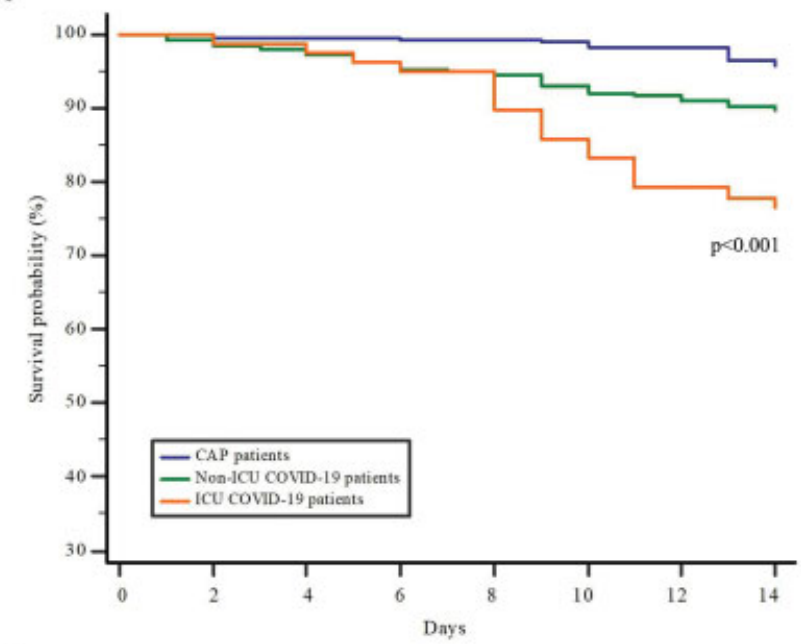

B

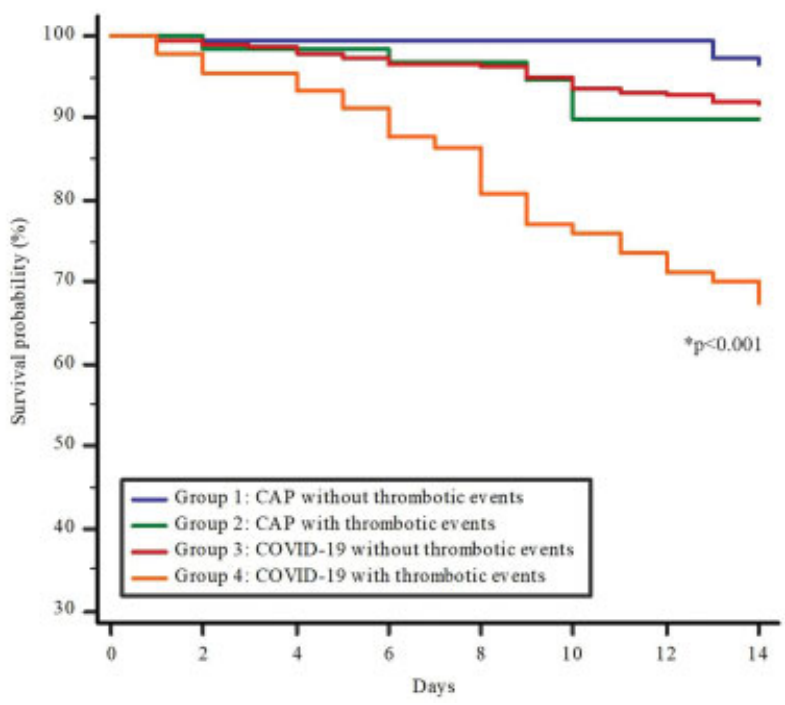

Fig. 1 Time to mortality in CAP and COVID-19 patients according to ICU admission (A) and in-hospital thrombotic events (B). * For group 1 versus group 2, 3, and 4; group 2 versus 4; group 3 versus 4. CAP, community-acquired pneumonia; ICU, intensive care unit.

when comparing CAP versus non-ICU COVID-19 (-Fig. 1). Age, male sex, chronic HF, COPD, CAD, and atherosclerotic risk factors such as hypertension and diabetes, and ICU admission were the clinical variables that distinguished survivors from nonsurvivor COVID-19 patients; among the laboratory variables WBC, hs-CRP, albumin, D-dimer, and sPO2 distinguished the two subgroups. Multiple regression analysis showed that thrombotic events were predictors of mortality in both CAP and COVID-19.

In COVID-19, pneumonia severity, as assessed by the CURB-65 score, heart failure, and serum albumin were also independent predictors of death. The CURB-65 score ${ }^{21}$ is a predictive marker for mortality in CAP and, in accordance with previous reports, ${ }^{23-25}$ could be also used to predict mortality in COVID-19 patients. It should be underscored, however, in the multivariable analysis that COVID-19 predicted mortality independently upon its clinical features. Data regarding the close relationship between albumin and mortality are consistent with a previous report from our 
Table 6 Patients' baseline characteristics according to mortality in CAP and COVID-19 patients

\begin{tabular}{|c|c|c|c|c|c|c|}
\hline & CAP patients & & & COVID patients & & \\
\hline & Survivors & Nonsurvivors & $p$-Value & Survivors & Nonsurvivors & $p$-Value \\
\hline No. & 542 & 17 & & 481 & 98 & \\
\hline Age & $71.6 \pm 16.2$ & $82.2 \pm 8.9$ & $<0.001$ & $63.2 \pm 15.6$ & $76.2 \pm 11.4$ & $<0.001$ \\
\hline Male sex & $62 \%$ & $47 \%$ & 0.212 & $54 \%$ & $65 \%$ & 0.044 \\
\hline ICU & n.a. & n.a. & n.a. & $9 \%$ & $37 \%$ & $<0.001$ \\
\hline Smoker & $20 \%$ & $12 \%$ & 0.386 & $16 \%$ & $16 \%$ & 0.579 \\
\hline CAD & $30 \%$ & $35 \%$ & 0.589 & $14 \%$ & $28 \%$ & 0.003 \\
\hline COPD & $32 \%$ & $47 \%$ & 0.193 & $10 \%$ & $22 \%$ & 0.006 \\
\hline Diabetes & $26 \%$ & $18 \%$ & 0.578 & $15 \%$ & $26 \%$ & 0.018 \\
\hline Hypertension & $72 \%$ & $76 \%$ & 0.789 & $47 \%$ & $61 \%$ & 0.015 \\
\hline Paroxysmal AF & $12 \%$ & $18 \%$ & 0.750 & $5 \%$ & $12 \%$ & 0.025 \\
\hline Chronic AF & $14 \%$ & $12 \%$ & 0.814 & $4 \%$ & $8 \%$ & 0.087 \\
\hline Chronic heart failure & $21 \%$ & $41 \%$ & 0.057 & $11 \%$ & $33 \%$ & $<0.001$ \\
\hline sPO2 (\%) & $93.4 \pm 6.9$ & $92.2 \pm 8.2$ & 0.918 & $94.7 \pm 5.9$ & $89.4 \pm 6.5$ & $<0.001$ \\
\hline WBC $\left(\mathrm{n} / \mathrm{mm}^{3}\right)$ & $9,860[7,410-13,470]$ & $12,880[9,360-14,240]$ & 0.069 & $5,900[4,430-7,970]$ & $7,180[4,840-9,465]$ & 0.027 \\
\hline $\operatorname{PLT}\left(\mathrm{n} / \mathrm{mm}^{3}\right) \times 10^{3}$ & 233 [179-300] & 255 [194-299] & 0.484 & 193 [159-246] & 189 [129-248] & 0.140 \\
\hline $\mathrm{Hs}-\mathrm{CRP}$ (mg/L) & $47[15-131]$ & 65 [21-93] & 0.911 & $45[18-117]$ & 90 [39-198] & $<0.001$ \\
\hline Albumin $(\mathrm{g} / \mathrm{L})$ & $35[31-38]$ & 31 [29-39] & 0.229 & $36[32-40]$ & $30[28-34]$ & $<0.001$ \\
\hline D-dimer (ng/mL) & 1188 [556-2392] & 982 [239-1684] & 0.293 & $879[445-1,920]$ & $1394[595-4,474]$ & 0.005 \\
\hline CURB65 score & $1.74 \pm 1.02$ & $2.41 \pm 0.94$ & 0.008 & $1.60 \pm 1.21$ & $2.74 \pm 0.88$ & $<0.001$ \\
\hline
\end{tabular}

Abbreviations: AF, atrial fibrillation; CAD, coronary artery disease; CAP, community-acquired pneumonia; COPD, chronic obstructive pulmonary disease, hs-CRP, high-sensitivity C-reactive protein; ICU, intensive care unit; OACs, oral anticoagulants; PLT, platelets; sPO2, oxygen saturation; WBC, white blood cell.

Note: Data are expressed as percentages, mean \pm SD, or median [interquartile range].

Table 7 Mortality in CAP and COVID-19 patients: COX regression analyses

\begin{tabular}{|c|c|c|c|c|}
\hline & \multirow[t]{2}{*}{ HR } & \multicolumn{2}{|c|}{$95 \% \mathrm{Cl}$} & \multirow[t]{2}{*}{$p$-Value } \\
\hline & & Lower & Upper & \\
\hline \multicolumn{5}{|l|}{ CAP'a } \\
\hline Thrombotic events & 4.29 & 1.60 & 11.52 & $<0.001$ \\
\hline \multicolumn{5}{|l|}{ COVID-19b } \\
\hline Thrombotic events & 2.15 & 1.38 & 3.31 & $<0.001$ \\
\hline Heart failure & 2.23 & 1.42 & 3.48 & $<0.001$ \\
\hline CURB- $65^{c}$ & 1.37 & 1.15 & 1.60 & $<0.001$ \\
\hline Albumin ${ }^{c}$ & 0.89 & 0.85 & 0.93 & $<0.001$ \\
\hline \multicolumn{5}{|l|}{ All patients ${ }^{\mathrm{b}}$} \\
\hline Thrombotic events & 2.58 & 1.75 & 3.79 & $<0.001$ \\
\hline CURB-65' & 1.70 & 1.41 & 2.06 & $<0.001$ \\
\hline Albumin ${ }^{c}$ & 0.88 & 0.85 & 0.92 & $<0.001$ \\
\hline Heart failure & 1.97 & 1.33 & 2.93 & $<0.001$ \\
\hline COVID-19 vs. CAP & 2.39 & 1.39 & 4.10 & 0.002 \\
\hline
\end{tabular}

Abbreviations: CAP, community-acquired pneumonia; $\mathrm{Cl}$, confidence interval; HR, hazard ratio.

after adjusting for the CURB-65 score.

${ }^{\mathrm{b}}$ After adjusting for age, sex, ICU admission, diabetes, arterial hypertension, coronary artery disease, heart failure, chronic obstructive pulmonary disease, atrial fibrillation, smoking habit, hs-CRP, D-dimer, creatinine, aspirin, anticoagulant use, and the recruitment centers.

${ }^{\mathrm{c}}$ For each increasing unit. 
group showing that serum album $<35 \mathrm{~g} / \mathrm{L}$ is associated with poor survival in COVID-19. ${ }^{26}$ The biological plausibility of this finding may be in the fact that albumin is an acute-phase reactant protein with anti-inflammatory, antioxidant, and anticoagulant properties; thus, in case of its consumption (as occurs in the case of acute or chronic inflammation) ${ }^{27}$ the body defense mechanisms are lowered. Further study is necessary to assess if normalization of serum albumin levels results in improving prognosis in COVID-19.

The study has implications and limitations. Compared with CAP, COVID-19 patients have a worse intrahospital clinical course as evidenced by the higher hospital length stay; furthermore, our CAP cohort did not need ICU treatment, thereby we cannot exclude a selection bias with an inclusion of less severe CAP. However, the difference in clinical outcomes persisted even when ICU COVID-19 patients were excluded from the analysis, reinforcing the hypothesis that CAP and COVID-19 have a different clinical course independently from disease severity. We cannot also exclude that the difference rate of thrombosis between CAP and COVID-19 could be even higher considering that at the beginning of pandemic the thrombotic complications in COVID-19 were likely underdiagnosed.

The typology of thrombosis (arterial in CAP, venous and arterial in COVID-19) suggests different mechanisms of disease and, perhaps, therapeutic approach. A retrospective study highlighted the favorable impact of anticoagulants in COVID-19 but the mortality was still elevated ${ }^{28}$; hence, inhibition of platelet function, which is also elevated in COVID-19, ${ }^{29}$ may be another option to be considered in future interventional trials. In this context, different options could be considered, such as the use of corticosteroids, which possess antiplatelet activity in vitro and in vivo ${ }^{30,31}$ or infusion of albumin, which inhibits platelet aggregation via an oxidative stress-mediated mechanism. ${ }^{32}$

We reported anticoagulation used as thromboprophylaxis at ward admission. Despite antithrombotic protocols were similar between hospitals in Italy, we have no data about preadmission thromboprophylaxis or changes on anticoagulation therapy that could occur during hospitalization; thus, we cannot evaluate if changes of anticoagulation could affect the thrombotic rate. Noteworthy, only few CAP patients were on heparin for thromboprophylaxis, while approximately $50 \%$ of COVID-19 patients were treated with heparin at admission. Thus, it is important to underlie that venous thrombosis was more common in COVID-19 despite the more extensive use of thromboprophylaxis.

Another limitation of the study is the lack of serial measurements of prothrombotic biomarkers that could provide more in-depth information about the pathobiology of thrombosis in CAP and COVID-19.

Finally, the study has been performed in a Caucasian population, thereby prospective study including also non-Caucasian population is needed to confirm the results.

In conclusion, the present study shows that, compared with CAP, thrombosis is more frequent in COVID-19 and associated with higher mortality risk. Early prediction of at-risk patients and identification of more appropriate antithrombotic treatment are needed to improve survival.

\section{What is known about this topic?}

- Patients with community-acquired pneumonia (CAP) and COVID-19 may experience thrombosis.

- Typology and incidence of thrombosis in the two settings are unclear.

\section{What does this paper add?}

- Patients with COVID-19 display a higher incidence of thrombosis compared with CAP (15.5 vs. 11.4\%, $p=0.046)$.

- In CAP patients, thrombosis occurs essentially in the arterial tree (11.4\%), while both venous thrombosis (8.3\%) and arterial thrombosis (7.2\%) are detectable in COVID-19.

\section{Funding}

This study was funded by Sapienza University of Rome (grant No. RM118164366B89BD to F.V.).

Conflict of Interest

None declared.

\section{References}

1 Bikdeli B, Madhavan MV, Jimenez D, et al; Global COVID-19 Thrombosis Collaborative Group, Endorsed by the ISTH, NATF, ESVM, and the IUA, Supported by the ESC Working Group on Pulmonary Circulation and Right Ventricular Function. COVID-19 and thrombotic or thromboembolic disease: implications for prevention, antithrombotic therapy, and follow-up: JACC stateof-the-art review. J Am Coll Cardiol 2020;75(23):2950-2973

2 Violi F, Pastori D, Cangemi R, Pignatelli P, Loffredo L. Hypercoagulation and antithrombotic treatment in coronavirus 2019: a new challenge. Thromb Haemost 2020;120(06):949-956

3 Katneni UK, Alexaki A, Hunt RC, et al. Coagulopathy and thrombosis as a result of severe COVID-19 infection: a microvascular focus. Thromb Haemost 2020;120(12):1668-1679

4 Al-Samkari H, Karp Leaf RS, Dzik WH, et al. COVID-19 and coagulation: bleeding and thrombotic manifestations of SARSCoV-2 infection. Blood 2020;136(04):489-500

5 Jain S, Self WH, Wunderink RG, et al; CDC EPIC Study Team. Community-acquired pneumonia requiring hospitalization among U.S. adults. N Engl J Med 2015;373(05):415-427

6 Pletz MW, Blasi F, Chalmers JD, et al. International perspective on the new 2019 American Thoracic Society/Infectious Diseases Society of America Community-Acquired Pneumonia Guideline: A Critical Appraisal by a Global Expert Panel. Chest 2020;158(05): 1912-1918

7 Cangemi R, Calvieri C, Falcone M, et al; SIXTUS Study Group. Relation of cardiac complications in the early phase of community-acquired pneumonia to long-term mortality and cardiovascular events. Am J Cardiol 2015;116(04):647-651

8 Violi F, Cangemi R, Falcone M, et al; SIXTUS (Thrombosis-Related Extrapulmonary Outcomes in Pneumonia) Study Group. Cardiovascular complications and short-term mortality risk in 
community-acquired pneumonia. Clin Infect Dis 2017;64(11): 1486-1493

9 Mei F, Fan J, Yuan J, et al. Comparison of venous thromboembolism risks between COVID-19 pneumonia and community-acquired pneumonia patients. Arterioscler Thromb Vasc Biol 2020;40(09): 2332-2337

10 Cangemi R, Casciaro M, Rossi E, et al; SIXTUS Study Group SIXTUS Study Group. Platelet activation is associated with myocardial infarction in patients with pneumonia. J Am Coll Cardiol 2014;64 (18):1917-1925

11 Falcone M, Venditti M, Shindo Y, Kollef MH. Healthcare-associated pneumonia: diagnostic criteria and distinction from communityacquired pneumonia. Int J Infect Dis 2011;15(08):e545-e550

12 Ye Z, Zhang Y, Wang Y, Huang Z, Song B. Chest CT manifestations of new coronavirus disease 2019 (COVID-19): a pictorial review. Eur Radiol 2020;30(08):4381-4389

13 Metlay JP, Waterer GW, Long AC, et al. Diagnosis and treatment of adults with community-acquired pneumonia. An official clinical practice guideline of the American Thoracic Society and Infectious Diseases Society of America. Am J Respir Crit Care Med 2019; 200(07):e45-e67

14 Konstantinides SV, Meyer G, Becattini C, et al; ESC Scientific Document Group. 2019 ESC guidelines for the diagnosis and management of acute pulmonary embolism developed in collaboration with the European Respiratory Society (ERS). Eur Heart J 2020;41(04):543-603

15 Thygesen K, Alpert JS, Jaffe AS, et al; Executive Group on behalf of the Joint European Society of Cardiology (ESC)/American College of Cardiology (ACC)/American Heart Association (AHA)/World Heart Federation (WHF) Task Force for the Universal Definition of Myocardial Infarction. Fourth universal definition of myocardial infarction (2018). Circulation 2018;138(20): e618-e651

16 Kernan WN, Ovbiagele B, Black HR, et al; American Heart Association Stroke Council, Council on Cardiovascular and Stroke Nursing, Council on Clinical Cardiology, and Council on Peripheral Vascular Disease. Guidelines for the prevention of stroke in patients with stroke and transient ischemic attack: a guideline for healthcare professionals from the American Heart Association/American Stroke Association. Stroke 2014;45(07): 2160-2236

17 Bonaca MP, Gutierrez JA, Creager MA, et al. Acute limb ischemia and outcomes with vorapaxar in patients with peripheral artery disease: results from the trial to assess the effects of vorapaxar in preventing heart attack and stroke in patients with atherosclerosis-thrombolysis in myocardial infarction 50 (TRA2 ${ }^{\circ} \mathrm{P}-\mathrm{TIMI} 50$ ). Circulation 2016;133(10):997-1005

18 Kearon C, Akl EA, Comerota AJ, et al. Antithrombotic therapy for VTE disease: Antithrombotic Therapy and Prevention of Thrombosis, 9th ed: American College of Chest Physicians EvidenceBased Clinical Practice Guidelines. Chest 2012;141(:2, Suppl): e419S-e496s
19 Loffredo L, Arienti V, Vidili G, et al; AURELIO Study Group. Low rate of intrahospital deep venous thrombosis in acutely ill medical patients: results from the AURELIO study. Mayo Clin Proc 2019;94(01):37-43

20 Hulshof AM, Brüggemann RAG, Mulder MMG, et al. Serial EXTEM, FIBTEM, and TPA rotational thromboelastometry observations in the Maastricht intensive care COVID cohort-persistence of hypercoagulability and hypofibrinolysis despite anticoagulation. Front Cardiovasc Med 2021;8:654174

21 Mulder MMG, Brandts L, Brüggemann RAG, et al. Serial markers of coagulation and inflammation and the occurrence of clinical pulmonary thromboembolism in mechanically ventilated patients with SARS-CoV-2 infection; the prospective Maastricht intensive care COVID cohort. Thromb J 2021;19(01):35

22 Santos-Gallego CG, Badimon JJ. The sum of two evils: pneumonia and myocardial infarction: is platelet activation the missing link? J Am Coll Cardiol 2014;64(18):1926-1928

23 Falcone M, Russo A, Cangemi R, et al. Lower mortality rate in elderly patients with community-onset pneumonia on treatment with aspirin. J Am Heart Assoc 2015;4(01):e001595

24 Ucan ES, Ozgen Alpaydin A, Ozuygur SS, et al; DEU COVID Study Group. Pneumonia severity indices predict prognosis in coronavirus disease-2019. Respir Med Res 2021;79:100826

25 Lazar Neto F, Marino LO, Torres A, et al; COVID Registry Team Medical Students Residents Attending physicians. Communityacquired pneumonia severity assessment tools in patients hospitalized with COVID-19: a validation and clinical applicability study. Clin Microbiol Infect 2021;27(07):1037.e1-1037.e8

26 Violi F, Cangemi R, Romiti GF, et al. Is albumin predictor of mortality in COVID-19? Antioxid Redox Signal 2021;35(02): 139-142

27 Ronit A, Kirkegaard-Klitbo DM, Dohlmann TL, et al. Plasma albumin and incident cardiovascular disease: results from the CGPS and an updated meta-analysis. Arterioscler Thromb Vasc Biol 2020;40(02):473-482

28 Tian $\mathrm{R}, \mathrm{Wu} \mathrm{W}$, Wang $\mathrm{C}$, et al. Clinical characteristics and survival analysis in critical and non-critical patients with COVID-19 in Wuhan, China: a single-center retrospective case control study. Sci Rep 2020;10(01):17524

29 Taus F, Salvagno G, Canè $S$, et al. Platelets promote thromboinflammation in SARS-CoV-2 pneumonia. Arterioscler Thromb Vasc Biol 2020;40(12):2975-2989

30 Cangemi R, Falcone M, Taliani G, et al; SIXTUS Study Group. Corticosteroid use and incident myocardial infarction in adults hospitalized for community-acquired pneumonia. Ann Am Thorac Soc 2019;16(01):91-98

31 Cangemi R, Carnevale R, Nocella C, et al; SIXTUS Study Group. Glucocorticoids impair platelet thromboxane biosynthesis in community-acquired pneumonia. Pharmacol Res 2018;131:66-74

32 Basili S, Carnevale R, Nocella C, et al; PRO -LIVER Collaborators. Serum albumin is inversely associated with portal vein thrombosis in cirrhosis. Hepatol Commun 2019;3(04):504-512 